\title{
Matérián
}

ISSN 1517-7076

Revista Matéria, v. 11, n. 3,pp. 260 - 266, 2006

http://www.materia.coppe.ufrj.br/sarra/artigos/artigo10813

\section{Efeitos de Diferentes Argilas Organofílicas nas Propriedades de Compósitos PET/Bentonita}

\author{
Itamara Farias Leite ${ }^{1}$, Claudia M. O. Raposo ${ }^{2}$, Laura Hecker Carvalho ${ }^{3}$, Suédina M. L. Silva ${ }^{3}$ \\ ${ }^{1} \mathrm{PPG}-\mathrm{CEMat} / \mathrm{CCT} / \mathrm{UFCG}$ \\ e-mail: itaflm@yahoo.com.br \\ ${ }^{2} \mathrm{DMG} / \mathrm{CCT} / \mathrm{UFCG}$ \\ e-mail: raposo@dmg.ufcg.edu.br \\ ${ }^{3} \mathrm{DEMa} / \mathrm{CCT} / \mathrm{UFCG}$ \\ Av. Aprígio Veloso, 882, 58109-970 - Bodocongó - Campina Grande/PB \\ e-mail: laura@dema.ufcg.edu.br, suedina@dema.ufcg.edu.br
}

\section{RESUMO}

Este trabalho tem por finalidade avaliar o efeito da utilização de duas bentonitas organofílicas, uma nacional, organofilizada com o sal brometo de cetil trimetil amônio, ASCM e outra importada comercial, Cloisite 30B nas propriedades de compósitos PET/bentonita pelo método de intercalação por fusão. Inicialmente, concentrados $(1: 1)$ de PET/bentonita foram preparados no misturador interno (Reomix 600) operando a $260{ }^{\circ} \mathrm{C} / 60 \mathrm{rpm} / 10 \mathrm{~min}$. Em seguida, os concentrados foram adicionados a matriz polimérica PET em quantidades necessárias para obtenção de $1-3 \%$ em massa de bentonita no compósito $\mathrm{PET} /$ bentonita para serem processadas em extrusora dupla-rosca contra-rotativa do reômetro de torque Haake empregando $275{ }^{\circ} \mathrm{C}$ e $60 \mathrm{rpm}$ e então injetadas em injetora Fluidmec a $260{ }^{\circ} \mathrm{C}$. Os compósitos foram denominados PET/ASCM e PET/CL e caracterizados por difração de raios-X (DRX), termogravimetria, (TGA) e propriedades tênseis e, as argilas, por espectroscopia no infravermelho com transformada de Fourier (FTIR), DRX e TGA. As análises de FTIR e DRX confirmaram a organofilização das argilas. A estabilidade térmica apresentou-se mais significativa para o compósito PET/ASCM e melhores propriedades tênseis foram verificadas para o compósito PET/CL com $3 \%$ em massa de Cloisite 30B.

Palavras chaves: PET, organofílica, propriedades tênseis.

\section{Effects of Different Organoclays on the Properties of PET/Bentonite Composites}

\section{ABSTRACT}

This work has for purpose evaluate the effect of the utilization of two organoclays bentonites, one national, organophilizated with the salt cetyl trimethyl ammonium bromide, ASCM and other commercial, Cloisite $30 \mathrm{~B}$ on the properties of the compounds PET/bentonite by melting intercalation. Initially, masterbatch (1:1) was added to PET so that PET/bentonita compounds with 1,2 and 3 weight \% of bentonite. These mixtures were extruded on a counter-rotating twin screw extruder coupled to the torque rheometer Haake operating at $275^{\circ} \mathrm{C}$ and $60 \mathrm{rpm}$ and then injection molded on a Fluidmec injection machine. The compounds were coded as PET/ASCM and PET/CL and were characterized by X rays diffraction, XRD, thermogravimetric analysis, TGA and tensile properties and, the clays infrared spectroscopy, FTIR, XRD and TGA. The analysis of FTIR and XRD confirmed the clay organophilization. The thermal stability was more significative for the compound PET/ASCM and the best tensile properties was verificated for the compound $\mathrm{PET} / \mathrm{CL}$ with 3 weight $\%$ of Cloisite $30 \mathrm{~B}$.

Keywords: PET, organoclays, tensile properties.

\section{INTRODUCÃO}

Nanocompósitos poliméricos são materiais híbridos em que substâncias inorgânicas de dimensões nanométricas são dispersas em uma matriz polimérica [1]. Um dos sistemas mais promissores é o híbrido 
baseado em polímeros orgânicos e argilominerais inorgânicos constituídos de silicatos em camadas [2]. Os nanocompósitos polímero/silicatos em camadas são similares aos compósitos poliméricos convencionais pois estes usam cargas para melhorar as propriedades não disponíveis nos polímeros não carregados. Entretanto, os nanocompósitos têm propriedades únicas quando comparadas aos polímeros, pois apresentam elevadas propriedades mecânicas, térmicas, de barreira, retardância de chama e estabilidade dimensional em baixos níveis de carregamento, tipicamente $1-5 \%$ em massa [프- $\underline{3}$.

Dentre os vários silicatos em camadas, as bentonitas têm sido uma das mais empregadas nos últimos anos como cargas para a preparação de nanocompósitos poliméricos por apresentar como argilomineral predominante a montmorilonita que, por sua vez, pertence ao grupo das esmectitas dioctaédricas e tem uma estrutura em camadas 2:1. Os espaçamentos entre as camadas são ocupados por cátions trocáveis, tipicamente $\mathrm{Na}^{+}$e/ou Ca ${ }^{2+}$, que balanceiam a deficiência de carga resultante da substituição isomórfica parcial de $\mathrm{Al}^{3+}$ por íons metálicos de menor valência, como por exemplo, o $\mathrm{Fe}^{2+}$. A troca iônica dos cátions entre as camadas por cátions orgânicos, particularmente íons alquilamônio, permite a modificação das superfícies, diminuindo a energia superficial e aumentando o espaçamento intercamada para intercalação do polímero [ㅁ-ㄱ]. Tendo em vista as propriedades apresentadas pelos nanocompósitos e as excelentes propriedades do PET, estudos têm sido realizados com o objetivo de desenvolver novas aplicações para este polímero e melhorar ainda mais as suas propriedades com a introdução de baixos percentuais em massa de argila.

\section{EXPERIMENTAL}

O poli(tereftalato de etileno) (PET), BG1180-W, grau garrafa, fornecido pela Braskem/BA sob a forma de pellets de coloração branca foi usado como matriz polimérica para a preparação de compósitos. E como carga utilizou-se uma bentonita denominada ASCN, fornecida pela Bentonit União Nordeste (BUN), Campina Grande/PB/Brasil, sob a forma de pó de granulometria inferior a $0,074 \mathrm{~mm}$. Esta bentonita foi organofilizada com o sal brometo de cetil trimetil amônio, Cetremide, fornecido pela Vetec. Empregou-se ainda como parâmetro comparativo, uma bentonita organofílica comercial denominada Cloisite 30B fornecida pela Southern Clay Products, Texas/EUA.

Organofilização da Carga: Para obter argilas organofílicas, $32 \mathrm{~g}$ de bentonita foram dispersas com $9,8 \mathrm{~g}$ do sal Cetremide em $1600 \mathrm{~mL}$ de água destilada a aproximadamente $80^{\circ} \mathrm{C}$ por 30 minutos sob agitação constante. Em seguida, o material foi colocado em repouso por $24 \mathrm{~h}$ a temperatura ambiente e então, filtrado e lavado com $2000 \mathrm{~mL}$ de água destilada para remover o excesso de brometo. Por fim, estes foram secos em estufa por $48 \mathrm{~h}$ a $60{ }^{\circ} \mathrm{C}$ e passadas em peneira ABNT \# 200. A bentonita ASCN depois de modificada organicamente foi designada por ASCM.

Preparação dos compósitos PET/bentonitas: para a obtenção dos compósitos, PET/bentonita, foram preparados concentrados (1:1) em um misturador interno acoplado ao reômetro de torque Haake System 90 operando a $260^{\circ} \mathrm{C}$ e com $60 \mathrm{rpm}$ por 10 minutos designados de PET/bentonita organofílica, PET/ASCM, e $\mathrm{PET} /$ bentonita organofílica comercial, PET/CL. Os concentrados obtidos foram adicionados à matriz polimérica, PET, em quantidades necessárias para obtenção de teores nominais de 1,2 e $3 \%$ em massa de bentonita. As misturas foram processadas em extrusora dupla rosca contra-rotativa acoplada ao reômetro de torque Haake utilizando temperatura de $275^{\circ} \mathrm{C}$ em todas as zonas de aquecimento e velocidade das roscas de $60 \mathrm{rpm}$. Posteriormente, as misturas foram injetadas em injetora Fluidmec a $260{ }^{\circ} \mathrm{C}$, na forma de corpos de prova de tração. Os compósitos PET/ASCM e PET/CL contendo 1, 2 e $3 \%$ em massa de bentonita foram denominados de PET/ASCM1, PET/ASCM2, PET/ASCM3, PET/CL1, PET/CL2 e PET/CL3 respectivamente.

Espectroscopia no Infravermelho: as análises no infravermelho das bentonitas foram realizadas em um espectrômetro AVATAR TM 360 ESP de Nicolet, com varredura de 4000 a $400 \mathrm{~cm}^{-1}$. As amostras de bentonitas foram caracterizadas na forma de pastilha feita a partir de 7,00 mg de argila e $0,100 \mathrm{~g}$ de $\mathrm{KBr}$ prensada a 5 toneladas por 30 segundos.

Difração de Raios X: as medidas de DRX foram realizadas em aparelho XRD-6000 Shimadzu utilizando-se radiação Cuk $\alpha$, tensão de $40 \mathrm{KV}$ e corrente de $30 \mathrm{~mA}$ com $2 \theta$ variando de 1,5 a $30^{\circ} \mathrm{e}$ velocidade de varredura de $0,02 \%$ min. Para a análise de DRX foram utilizadas cargas modificadas organicamente sob a forma de pó com granulometria inferior a $0,074 \mathrm{~mm}$ e para os compósitos PET/bentonita esta análise foi realizada em corpos de prova moldados por injeção.

Análise Termogravimétrica: as análises de TGA foram conduzidas em aparelho TGAA S1H da Shimadzu empregando taxa de aquecimento de $10{ }^{\circ} \mathrm{C} / \mathrm{min}$ e temperatura de 25 a $900{ }^{\circ} \mathrm{C}$ sob atmosfera de nitrogênio. Para realização desta análise utilizou-se amostras retiradas de corpos de prova para ensaios tênseis.

Propriedades Tênseis: os compósitos de PET/bentonita foram caracterizados mecanicamente em tração segundo as normas ASTM D638. Estes ensaios foram realizados a temperatura ambiente usando-se uma máquina universal de ensaios Lloyd LR-10KN empregando-se uma taxa de deslocamento de $4 \mathrm{~mm} / \mathrm{min}$. 
Os valores reportados para as propriedades mecânicas são valores médios obtidos a partir pelo menos 5 corpos de prova.

\section{RESULTADOS E DISCUSSÃO}

Os espectros no infravermelho do sal Cetremide (a), ASCM (b) e Cloisite 30B (c) são apresentados na Figura 1. O sal orgânico, Cetremide usado na organofilização da bentonita ASCM apresenta uma banda de estiramento C-H em $3020 \mathrm{~cm}^{-1}$ característica dos cátions alquil amônio. As bandas em 2920 e $2853 \mathrm{~cm}^{-1}$ para a ASCM, e em 2920 e $2849 \mathrm{~cm}^{-1}$ para a Cloisite 30B, são correspondentes aos modos de vibração assimétrico e simétrico do grupo $\mathrm{CH}_{2}$ respectivamente [요밀. Em 1479 e $1464 \mathrm{~cm}^{-1}$ são caracterizadas as deformações assimétricas dos grupos $\mathrm{CH}_{3}$ e $\mathrm{CH}_{2}$ indicando a presença do surfactante nas argilas ASCM e Cloisite 30B [9].

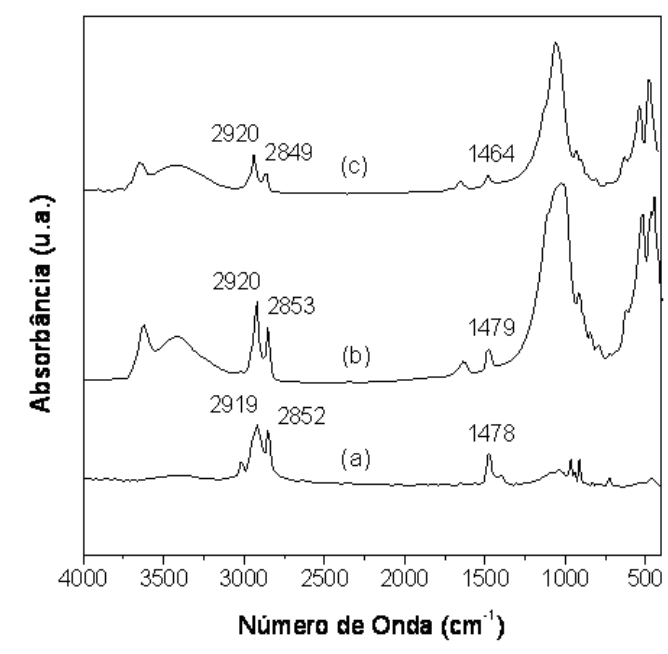

Figura 1: Espectros no infravermelho do sal Cetremide (a), argila organofílica local ASCM (b), e argila organofílica comercial Cloisite $30 \mathrm{~B}(\mathrm{c})$.

Os difratogramas da bentonita natural (ASCN), modificada organicamente (ASCM) e organofílica comercial (Cloisite 30B), são apresentados na Figura 2. Os dados de DRX da argila ASCM mostram um

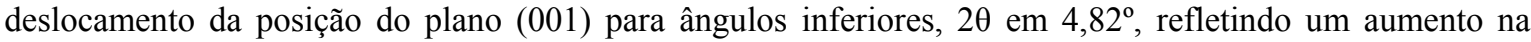
distância basal, $\mathrm{d}_{(001)}$, de 1,3 $\mathrm{nm}(\mathrm{ASCN})$ para 1,8 $\mathrm{nm}(\mathrm{ASCM})$ [11]. Estes dados confirmam a troca iônica no espaço entre as camadas da argila e, portanto, a obtenção de argilas organofílicas [12]. O espaço interlamelar $\mathrm{d}_{(001)}$, das argilas ASCM e Cloisite 30B são equivalentes a 1,8 nm. Dessa forma, observa-se que, mesmo tendo utilizado distintas argilas e diferentes surfactantes estas apresentam-se semelhantes em relação ao $\mathrm{d}_{(001)}$. Tal fato sugere que o incremento do espaçamento interlamelar basal das argilas organofílicas baseia-se no modo como a mesma se orienta entre as lamelas do argilomineral [13].

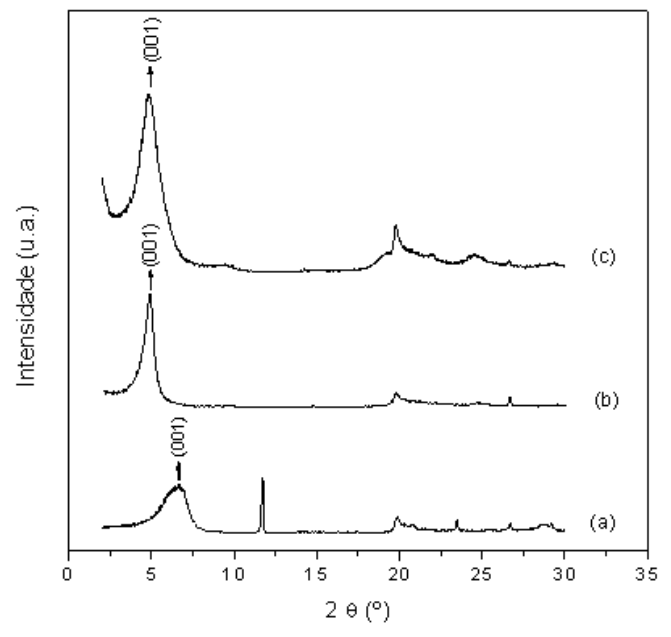

Figura 2: Difratogramas das bentonitas ASCN (a), ASCM (b) e Cloisite 30B (c). 
Os difratogramas do PET puro e dos compósitos PET/ASCM e PET/CL contendo 1, 2 e $3 \%$ em massa de argila são apresentados na Figura 3. Estes dados indicam que em todos os casos os espaçamentos interlamelares basais, $\mathrm{d}_{(001)}$, das argilas nos compósitos PET/bentonita apresentam-se reduzidos depois da incorporação do polímero. Analisando os compósitos contendo 1, 2 e $3 \%$ em massa de argilas organofílicas, ASCM e Cloisite $30 \mathrm{~B}$, observam-se resultados similares com $\mathrm{d}_{(001)}$ de 1,5 $\mathrm{nm}$. No entanto, sugere-se que não há formação de um nanocompósito, uma vez que o espaçamento basal das organofílicas nos compósitos PET/ASCM e PET/CL são inferiores ao $\mathrm{d}_{(001)}$ de ambas argilas, ASCM e Cloisite $30 \mathrm{~B}$, cujos espaçamentos interplanares são semelhantes a 1,8 nm. Assim, pode-se dizer que os resultados de DRX para os compósitos com argila organofílica, PET/ASCM e PET/CL, apresentam uma morfologia de microcompósito.

Acredita-se que a afinidade carga/matriz seja essencial para se obter nanocompósitos intercalados e/ou esfoliados e que o aumento da polaridade da superfície da argila aumenta a compatibilidade com a matriz polimérica polar [14-18]. Isto não foi verificado neste estudo, nem mesmo para os compósitos PET/CL onde, apesar de tanto a argila quanto o polímero apresentarem grupos polares, a obtenção de nanocompósitos não foi evidenciada. É provável que as condições de processamento adotadas tenham sido o fator limitante para a obtenção de nanocompósitos PET/CL.
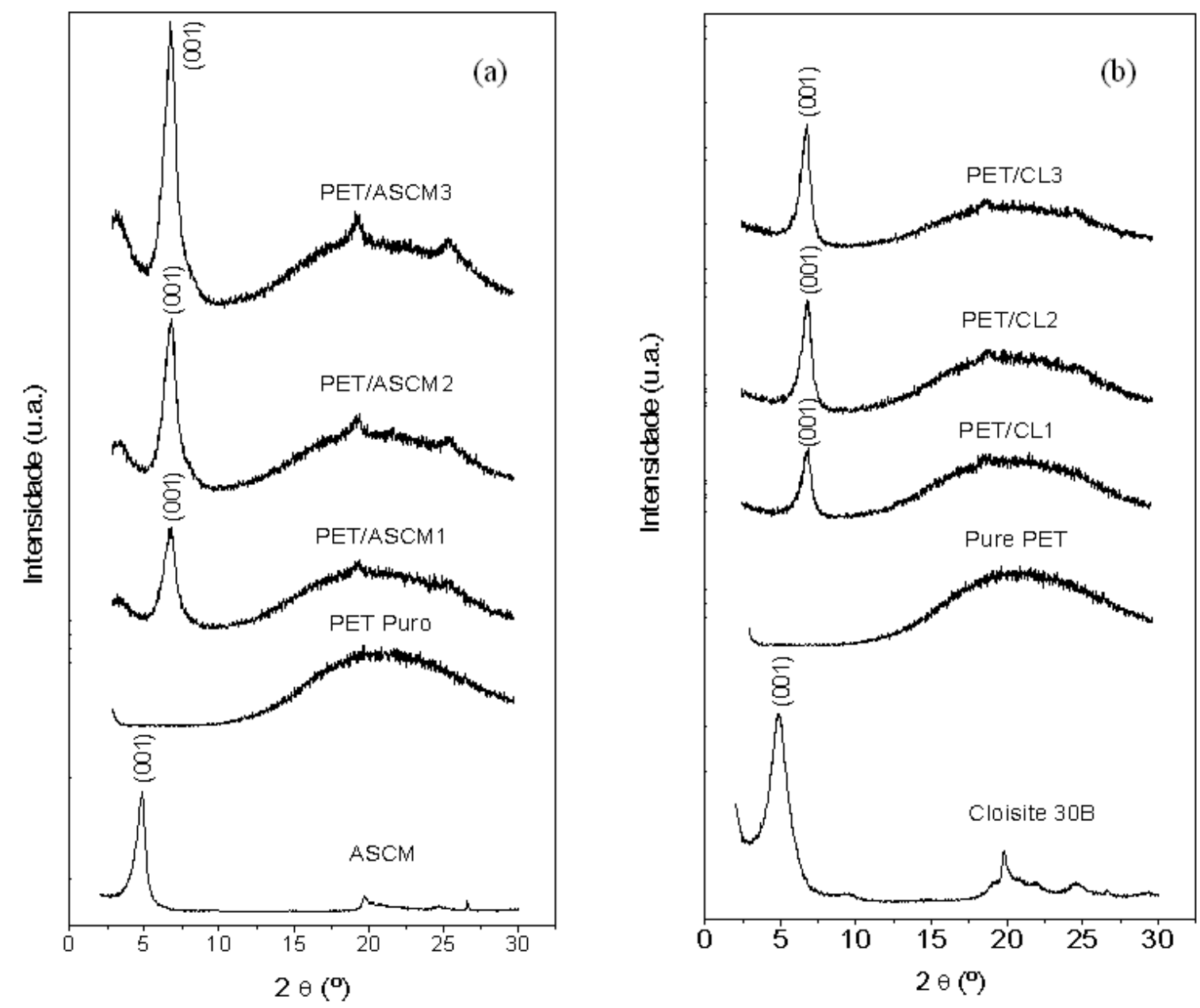

Figura 3: Difratogramas do PET puro e dos compósitos PET/ASCM (a) e PET/CL (b) contendo 1, 2 e $3 \%$ em massa de argila.

Na Tabela 1 e Figura 4 estão apresentados os resultados das análises termogravimétricas do PET puro e dos compósitos PET/ASCM e PET/CL contendo 1, 2 e $3 \%$ em massa de argila respectivamente. Analisando os compósitos contendo 1, 2 e $3 \%$ em massa de argilas organofílicas, PET/ASCM e PET/CL, as temperaturas $\mathrm{T}_{\mathrm{Di}}$ 's são de $375,369,358,332,352$ e $326^{\circ} \mathrm{C}$ respectivamente, também inferiores àquela apresentada pelo PET puro. Observa-se através destes dados que o compósito PET/CL, apresenta-se menos estável a decomposição que o compósito PET/ASCM. Isto pode ser devido à decomposição do sal MT2EtOH utilizado na organofilização da argila Cloisite 30B durante as etapas de processamento. A temperatura de 
fusão do sal MT2EtOH é de $200{ }^{\circ} \mathrm{C}$, ou seja, inferior a do sal Cetremide, que fica em torno de $243{ }^{\circ} \mathrm{C}$, o que pode ter acelerado a degradação do PET em elevadas temperaturas [19]. Nota-se também uma segunda etapa de decomposição relativa ao restante do material orgânico nos compósitos PET/ASCM e PET/CL. A preparação dos compósitos de PET intercalados por fusão necessita de elevadas temperaturas podendo não apenas alterar a interface entre a carga e a matriz, mas também induzir a degradação do poliéster como descrito por Lee et al. [20]. O $\mathrm{T}_{\mathrm{Max}}$ representa os valores máximos de decomposição dos corpos de prova obtidos da derivada nas curvas termogravimétricas.

Tabela 1: Valores das temperaturas de decomposição do PET puro e dos compósitos PET/ASCM e PET/CL com 1,2 e $3 \%$ em massa de argila.

\begin{tabular}{|c|c|c|c|}
\hline \multirow{2}{*}{ Compósitos } & \multicolumn{2}{|c|}{$\begin{array}{c}1^{\circ} \text { Etapa } \\
\text { Decomposição }\end{array}$} & \multirow{2}{*}{$\begin{array}{c}2^{\circ} \text { Etapa } \\
\text { Decomposição } \\
T_{\mathrm{Di}} \\
\left({ }^{\circ} \mathbf{C}\right)\end{array}$} \\
\hline & $\begin{array}{l}\mathbf{T}_{\mathrm{Di}} \\
\left({ }^{\circ} \mathrm{C}\right)\end{array}$ & $\begin{array}{l}\mathbf{T}_{\text {Max }} \\
\left({ }^{\circ} \mathrm{C}\right)\end{array}$ & \\
\hline PET Puro & 374,7 & 482,8 & $\begin{array}{l}---- \\
--1\end{array}$ \\
\hline PET/ASCM1 & 375,1 & 486,9 & 588,1 \\
\hline PET/ASCM2 & 369,2 & 486,8 & 619,8 \\
\hline PET/ASCM3 & 358,9 & 475,9 & 511,9 \\
\hline PET/CL1 & 332,0 & 469,5 & 535,3 \\
\hline PET/CL2 & 352,5 & 477,8 & 569,5 \\
\hline PET/CL3 & 326,2 & 469,5 & 535,3 \\
\hline
\end{tabular}
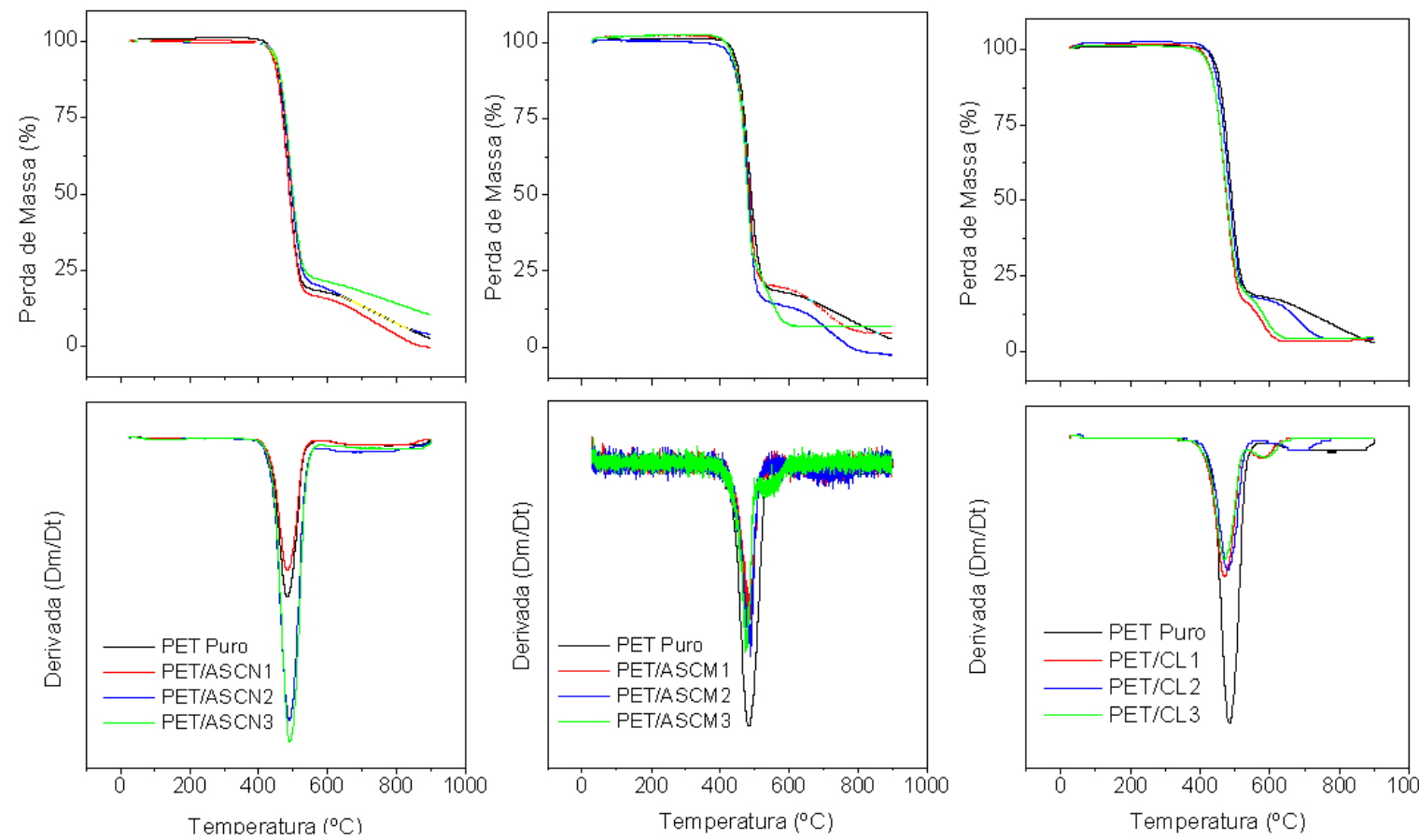

Figura 4: Análises termogravimétricas do PET puro e dos compóstos PET/ASCN (a) e PET/ASCM (b) e PET/CL (c) contendo 1, 2 e $3 \%$ em massa de argila.

Os resultados das propriedades mecânicas de resistência a tração $(\sigma)$ e módulo de Young (E) do PET puro e dos compósitos PET/ASCM e PET/CL com 1, 2 e $3 \%$ em massa de argila são apresentados na Tabela 2. 
Em se tratando de argilas organofílicas vale salientar que a compatibilidade da organofílica com a matriz polimérica é também de grande importância para formar um nanocompósito [19]. Analisando o compósito PET/ASCM nota-se que os valores de $\sigma$ apresentam-se similares aos obtidos pelo compósito $\mathrm{PET} / \mathrm{CL}$ e que o E apresenta discreto aumento para o compósito contendo $3 \% \mathrm{em}$ massa de argila ASCM, $\mathrm{PET} / \mathrm{ASCM}$. Este fato pode estar possivelmente associado a um aumento na cristalinidade favorecida pelo processo de injeção, como confirmado por DRX, assim como do efeito reforçante dos cristais da argila [18]. Os dados de tração reportados foram resultados médios de no mínimo 6 corpos de prova por composição.

Tabela 2: Valores das propriedades mecânicas do PET puro e dos compósitos PET/ASCM e PET/CL com 1, 2 e $3 \%$ em massa de argila.

\begin{tabular}{ccccc}
\hline Amostras & $\begin{array}{c}\boldsymbol{\sigma} \\
(\mathbf{M P a})\end{array}$ & $\Delta \%$ & $\begin{array}{c}\mathbf{E} \\
(\mathbf{M P a})\end{array}$ & $\Delta \%$ \\
\hline PET Puro & $46,9 \pm 0,6$ & - & $1859,3 \pm 70,6$ & - \\
PET/ASCM1 & $46,2 \pm 0,1$ & 1,40 & $1909,6 \pm 62,7$ & 2,7 \\
PET/ASCM2 & $47,9 \pm 1,1$ & 2,14 & $1906,7 \pm 84,6$ & 2,5 \\
PET/ASCM3 & $48,4 \pm 0,4$ & 3,3 & $2004,7 \pm 35,3$ & 7,8 \\
PET/CL1 & $45,9 \pm 1,7$ & 2,1 & $1923,1 \pm 30,5$ & 3,4 \\
PET/CL2 & $46,2 \pm 0,9$ & 1,4 & $1940,1 \pm 19,7$ & 4,3 \\
PET/CL3 & $48,4 \pm 1,4$ & 3,2 & $1965,6 \pm 14,3$ & 5,7 \\
\hline
\end{tabular}

\section{CONCLUSÃO}

Os dados de FTIR conjuntamente com os difratogramas confirmaram a obtenção da argila organofílica, ASCM. O DRX para os compósitos PET/ASCM e PET/CL contendo $1-3 \%$ em massa de bentonita apresentaram espaçamentos basais equivalentes a $1,5 \mathrm{~nm}$ e, portanto, a obtenção de materiais com estrutura de microcompósito. Os resultados de TGA dos compósitos PET/ASCM mostraram-se mais estáveis termicamente do que as amostras, PET/CL. As propriedades de resistência à tração para ambos sistemas apresentaram-se similares e o módulo de Young mostrou discreto aumento para o compósito, PET/ASCM3.

\section{AGRADECIMENTOS}

Os autores agradecem a Bentonit União do Nordeste - BUN e a Braskem pela doação dos materiais. A RENAMI e a CAPES pelo apoio financeiro.

\section{REFERÊNCIAS}

[1] WANG, K.H., CHOI, M.H., KOO, C.M., CHOI, Y.S., CHUNG, I.J., "Synthesis and Characterization of Maleated polyethylene/clays Nanocomposites”, Polymer, v. 42, pp. 9819-9826, 2001.

[2] TIDJANI, A., WILKIE C.A., "Photo-oxidation of Polymeric - Inorganic Nanocomposite: Chemical, Thermal Stability and Fire Retardancy Investigation”, Polymer Degradation and Stability, v. 74, pp. 33-37, 2001.

[3] AWAD, W.H., GILMAN, J.W., NYDEN, M., HARRIS, R.H., SUTTO, T.E., CALLAHAN, J., TRUlOVE, P.C., DELONG, H.C., FOX, D.M., "Thermal Degradation Studies of alkylimidazolium Salts and their Application in Nanocomposites", Thermochimica Acta, v. 409, pp. 3-11, 2004. 
[4] CHANG, J., KIM, S.J., JOO, Y.L., IM, S., "Poly(ethylene terephathalate) Nanocomposites by in Situ Interlayer Polymerization: the thermo-mechanical Properties and Morphology of the Hybrid Fibers", Polymer, v. 45, pp. 919-926, 2004.

[5] XIAO, J., HU, Y., WANG, Z., TANG, Y., CHEN, Z., FAN, W., "Preparation and Characterization of poly(butylenes terephthalate) Nanocomposites from Thermally Stable organic-modified Montmorillonite”, European Polymer Journal, v. 41, pp. 1030-1035, 2005.

[6] QIN, H., ZHAO, C., ZHANG, S., CHEN, G., YANG, M., "Photo-oxidative Degradation of polyethylene/montmorillonite Nanocomposite", Polymer Degradation and Stability, v. 81, pp. 497 $500,2003$.

[7] WAN, C., QIAO, X., ZHANG, Y., ZHANG, Y., "Effect of Different Clay Treatment on Morphology and Mechanical Properties of PVC-clay Nanocomposites”, Polymer Testing, v. 22, pp. 453-461, 2003.

[8] MADEJOVÁ, J,. "FTIR Techniques in Clay Mineral Studies”, Vibrational Spectroscopy, v. 31, pp. 1-10, 2003.

[9] KOZAK, M., DOMKA, L., "Adsorption of the Quaternary Ammonium Salts on Montmorillonite", Journal of Physics of Solids, v. 65, pp. 441-445, 2004.

[10] XI, Y., DING, Z., HE, H., FROST, R.L., "Infrared Spectroscopy of Organoclays Synthesized with the Surfactant Octadecyltrimethylammonium Bromide", Spectrochimica Acta Part A, v. 61, pp. 515525,2005 .

[11] OLPHEN, H.V., An Introduction to Clay Colloid Chemistry, ed. 2, Krieger Publishing Company, Malabar, Florida, 1991.

[12] RODRÍGUEZ-SARMIENTO, D.C., PINZÓN-BELLO, “Adsorption of Sodium Dodecylbenzene Sulfonate on Organophilic Bentonites”, Applied Clay Science, v. 18, pp. 173-181, 2001.

[13] FU, X.A., QUTUBUDDIN, S., "Swelling Behavior of Organoclays in Styrene and Exfoliation in Nanocomposites", Journal of Colloid and Interface Science, v. 283, pp. 373-379, 2005.

[14] CHO, J., PARK, D. R., “Nylon 6 Nanocomposites by melt compounding”, Polymer, v. 42, pp. 10831094, 2001.

[15] DENNIS, H.R., HUNTER, D.L., CHANG, D., KIM, S., WHITE, J.L., CHO, J.W., PAUL, D.R., "Effect of Melt Processing Conditions on the Extend of Exfoliation in organoclay-based Nanocomposites", Polymer, v. 42, pp. 9513-9522, 2001.

[16] LEE, K.M., HAN, C.D., "Rheology of Organoclay Nanocomposites, Effects of Polymer Matrixorganoclay Compatibility and the Gallery Distance of Organoclay”, Macromolecules, v. 36, pp. 7165-7178, 2003.

[17] LEE, K.M., HAN, C.D., "Effect of Hydrogen Bonding on the Rheology of polycarbonate / organoclay Nanocomposites", Polymer, v. 44, pp. 4573-4588, 2003.

[18] SÁNCHEZ-SOLÍS, A., ROMERO-IBARRA, I., ESTRADA, M.R., CELDERAS, F., MANERO, O., "Mechanical and Rheological Studies on Polyrthylene terephthalate-montmorillonite Nanocomposites", Polymer Engineering and Science, v. 44, pp. 1094-1102, 2004.

[19] GUAN, G., Li, C., ZHANG, D., "Spinning and Properties of poly(ethylene terephthalate) /organomontmorillonite Nanocomposite Fibers", Journal of Applied Polymer Science, v. 95, pp. 1443-1447, 2005.

[20] LEE, S., MA, Y.T., RHEE, H., KIM, J., "Exfoliation of Layered Silicate Facilitated by ring-opening Reaction of Cyclic Oligomers in PET-clay Nanocomposites”, Polymer, v. 43, pp. 2201-2210, 2005. 